\title{
Medición científica entre la intervención y la representación
}

Gilda Macedo Mira Andreu Egresada Universidad Autónoma Metropolitana

Resumen

Se analiza la propuesta empirista de van Fraassen en torno a la medición científica considerando las objeciones hechas a las teorías representacionalistas de la medición, para argumentar que la medición es un proceso complejo iterativo en el que teoría y experimentación se complementan y retroalimentan en cada etapa o fase.

Palabras clave: medición científica, representación, empirismo, experimento, proceso.

\section{Abstract}

Analysis of van Fraassen's empirist aproach of scientific measurement considering the objections of the Representational Theory of Measurement, to make the argument about measurement as a iterative complex process where theory and experimentation complement each other and feed back in each stage or phase.

Key words: Scientific Measurement, Representation, Empiricism, Experiment, Process. 


\section{Introducción}

$\mathrm{Z}^{\text {l tema de la representación científica en la filosofía de la cien- }}$ cia perdió cierto interés hacia finales de la década de 1980 dando mayor peso al de las prácticas científicas. Uno de los principales factores que contribuyeron a colocar en segundo plano a la representación científica fue la obra de Ian Hacking Representing and Intervening (1983), en donde se sugiere fuertemente sustituir la representación por la intervención como principal elemento para argumentar a favor de un realismo de entidades. Para este autor el experimento desempeña un papel central en la ciencia y los resultados obtenidos proveen de evidencia científica y tienen la función fundamental de proveer buenas razones para creer en las entidades que postulan las teorías. En los procesos experimentales la medición científica cumple un papel importante al asignar numerales a las cantidades porque de esta forma se obtiene un valor fijo por medio de la manipulación e intervención. Si bien los ejemplos pertinentes a la intervención, que son utilizados por Hacking, se centran en los microscopios y la observación a través de los mismos, en dicho libro también hay un apartado sobre la medición en el que discute aspectos que pertenecen a la racionalización, considerándola en el lado de la conceptualización y no de la experimentación. Sin embargo, el presente texto argumentará que la medición es una actividad científica que involucra tanto elementos abstractos y racionales como experimentales en sus procesos.

Por otro lado, Van Fraassen quien ha defendido una postura opuesta al realismo de entidades de Hacking, desde la misma época estableció una postura empirista que se inclina en favor de la representación. El libro Scientific Representation: Paradoxes of Perspective, de van Fraassen (2008) ha reactivado la discusión filosófica en torno al tema de la representación científica y uno de los elementos centrales que ha integrado en su formulación es la noción de me- 
dición cientifica como una actividad central en la construcción de representaciones en la ciencia. Esta inclusión es un aporte novedoso al empirismo que van Fraassen ha trabajado desde la década de 1980. El interesante artículo de Vadim Batitsky (1998) sirve de antecedente a dicha inclusión porque critica la postura de van Fraassen y sostiene que las interpretaciones empiristas de la relación entre las teorías y el mundo enfrentan dificultades insuperables cuando se considera la relación entre teorías científicas cuantitativas y el mundo. Batitsky plantea de manera convincente que el vínculo entre teorías científicas cuantitativas, es decir aquellas que han desarrollado ciencias más avanzadas, y el mundo se establece por medio de mediciones científicas; por ello, las interacciones con el mundo que conducen a una sistemática asignación de números a ciertos atributos físicos se dan a través de la medición y no principalmente mediante la observación simple, tal y como van Fraassen sugería. El punto de Batitsky es que van Fraassen, a pesar de que habla de medición científica en The Scientific Image (1980), sólo le otorga un papel secundario colocando a la observación como central.

Ahora, en su libro Scientific Representation, van Fraassen le otorga un mayor peso y funciones más complejas a su idea de medición científica respecto a la que aparece en La imagen cientifica, posiblemente en respuesta a las críticas de Batitsky. En términos generales, ésta es una práctica que genera representaciones mediante una actividad compleja que integra aparatos, matrices experimentales, cálculos matemáticos, conceptos teóricos, teorías, modelos, y cuya principal finalidad es asignar números a magnitudes. Cuando un número es asignado a una magnitud, según la teoría representacionalista de la medición, es interpretado como una representación de dicha magnitud. Una magnitud es entendida, en este contexto, como una propiedad medible de un sistema físico, lo cual significa que se le pueden asignar distintos valores como resultado de 
procesos de medición. Van Fraassen incorpora en su empirismo estructural una peculiar caracterización de medición científica que consiste en integrar tres elementos, a saber, el sujeto que mide, el instrumento mediante el cual se mide y la teoría científica involucrada en la medición. $Y$ es peculiar, entre otras cosas, porque a diferencia de teorías representacionalistas de la medición como la de Suppes (Scott y Suppes, 1957), que incluye modelos, la de van Fraassen además incorpora un modelo que él denomina "de superficie", el cual consiste en integrar tanto el tradicional modelo de datos con registros de observaciones llevadas a cabo meticulosamente por largos periodos de tiempo. La idea de medición que desarrolla van Fraassen en Scientific Representation está arraigada en lo que genéricamente se conoce como teoría representacionalista de la medición, esta postura privilegia los aspectos teóricos de la medición.

El presente texto argumentará en favor de una concepción de la medición como un proceso que involucra tanto aspectos teóricos representacionalistas como aspectos prácticos experimentales. Para ello se analizarán los elementos centrales que definen una postura representacionalista de la medición partiendo de la propuesta más reciente que es la de van Fraassen y sus antecedentes, así como algunas de las dificultades que enfrenta dicho enfoque. Se analizará un caso histórico, el experimento de Albert A. Michelson (18521931) de medición de la velocidad de la luz entre dos puntos relativamente inmóviles en el vacío realizado en 1877, para apoyar la idea de un proceso de medición en el que se interrelacionan tanto aspectos teóricos como experimentales. Lo anterior para concluir que la medición es un proceso complejo que produce productos, es decir, representaciones, en el que la racionalidad y la experimentación se vinculan e interrelacionan de manera iterativa apoyándome en Hasok Chang (2006) quien sostiene la noción de proceso como una "iteración epistémica" la cual consiste en que, al partir 
de una idea provisional respecto de la naturaleza de un proceso, se genera una teoría que modifica parcial y sustantivamente a la idea original. Y esta modificación a su vez generará otra idea que servirá de apoyo para continuar este proceso de retroalimentación.

\section{Van Fraassen y su postura representacionalista de la medición}

Las teorías representacionalistas de la medición sostienen, en términos generales, que la medición es una representación numérica que asigna números a cantidades; desde esta visión, es un mapeo entre elementos (cosas, objetos) de una clase a elementos (cosas, objetos) de otra. El enfoque representacionalista interpreta a la medición como la representación numérica de las propiedades medidas. Entre aquellos que adoptaron este enfoque destacan: Russell (1903), Campbell (1920), Nagel (1932), Stevens (1946, 1951), Suppes y Zinnes (1963) y Krantz et al. (1971). Van Frassen elabora una postura representacionalista estructuralista de la medición que asume una interpretación semántica de las teorías apoyada en la tradición heredada de Patrick Suppes.

El empirismo estructural de van Fraassen propone una visión empirista del estructuralismo en la filosofía de la ciencia. Sostiene que no es suficiente comprender las teorías y los modelos para entender la representación científica; para abarcar la representación científica y la representación teórica hay que incluir la medición y la experimentación, ya que éstos son medios para representar científicamente aquello que depende directamente de la teoría. La representación científica está mediada por la medición y la experimentación, y ambas actividades científicas (prácticas científicas) son teóricamente dependientes. En otras palabras, la medición científica, la cual usualmente se hace mediante instrumentos y/o experimentos, media entre los datos observacionales y las teorías. 
Expresado en el esquema estándar que utiliza la filosofía de la ciencia podríamos decir que en el nivel inferior están los datos observacionales mientras que en el superior están las teorías, mientras que las mediciones estarían en un nivel intermedio entre ambos extremos. El conocimiento científico generado por las mediciones científicas no consiste en solamente datos observacionales y tampoco en teorías científicas con poder explicativo, sino que está entre estos dos tipos de conocimiento. Para van Fraassen, la representación científica no se agota en un estudio exhaustivo del papel que desempeñan las teorías o los modelos teóricos; es por eso que para tener una comprensión más completa de la medición científica se la debe contemplar tanto en sus características instrumentales como en sus aspectos generales. La tesis que el autor defiende a lo largo de su mencionado libro es que medir, al igual que teorizar, es representar, ya que la medición localiza su objetivo en un espacio construido teóricamente.

El empirismo estructural de van Fraassen analiza la medición científica en torno a la relación que las teorías tienen con el mundo físico y/o los fenómenos. Es expuesta por el autor como una actividad central junto con la experimentación al construir representaciones científicas por medio de modelos científicos. El proceso de medir relaciona tres tipos de modelos (de datos, de superficie y el teórico) e involucra tres elementos (instrumento, agente y marco teórico). El sujeto (agente) está familiarizado con el instrumento de medición, en muchos casos diseñado/construido particularmente para medir cierta cantidad que está fundamentada en teorías científicas (marco teórico). Los modelos de datos se afinan y analizan para configurar el modelo de superficie, es decir, el que representa las estructuras del fenómeno, evento o proceso que isomórficamente encaja en el modelo teórico.

Tradicionalmente se entiende a los modelos científicos como descripciones interpretativas de un fenómeno que facilitan el ac- 
ceso al mismo. El acceso puede ser perceptual, involucrando los sentidos (generalmente la observación), y/o intelectual. Cuando el acceso no es perceptual, es por visualización. Las descripciones interpretativas pueden o no apoyarse en idealizaciones, simplificaciones o analogías a descripciones interpretativas de otros fenómenos. Los modelos pueden ser objetos, como un avión de juguete, o ser teóricos, entidades abstractas, como el modelo standard de la estructura de las materia y sus partículas fundamentales (BailerJones, 2009: 1-2).

De acuerdo con van Fraassen, los modelos científicos con frecuencia contienen muchos más elementos que no corresponden a ningún rasgo observable en el dominio; es decir, el modelo no puede establecer un mapeo entre los aspectos observables y los no observables. Para su proyecto empirista es muy importante que los modelos científicos representen las estructuras de los fenómenos observables. El modelo es -desde su perspectiva- útil (práctica y teóricamente) para pensar los fenómenos ajustados a una estructura observable mayor. Van Fraassen quiere caracterizar la representación científica desde la medición y el modelaje para mostrar cómo son representados por la ciencia los fenómenos observables. En este intento, comprende que tanto la medición como los modelos científicos tienen un carácter teórico y otro práctico, ambos dentro de un contexto histórico. La construcción de modelos científicos por medio de la medición científica depende del contexto y está dictada por la teoría de fondo que ofrece una dimensión espacial en donde las representaciones científicas se producen.

Si bien van Fraassen está consciente del valor histórico en los procesos de medición e intenta ver a la medición tanto desde sus procesos internos como desde sus aspectos más teóricos, reconoce que tanto la medición científica como el modelaje científico son, en sus núcleos, principalmente abstractos y matemáticos. De ahí surge la relevancia para su empirismo estructuralista al intentar 
entender cómo la medición y el modelaje pueden establecer bases empíricas de los fenómenos observables.

Sin embargo, la caracterización de van Fraassen resulta insuficiente para estudiar a la medición científica dominantemente como producto más que como proceso, porque un análisis en torno a la medición no se limita exclusivamente a los productos de la medición sino a todo el proceso que hace posible dichos productos. Si bien es correcto colocar a la medición científica entre los datos y las teorías, siguiendo el esquema tradicional tal como lo hace, ello no permite concebir en toda su complejidad la interrelación de procesos necesaria para generación de conocimiento científico a través de prácticas de medición. Como, por ejemplo, la temperatura que requirió un largo proceso de medición que duró aproximadamente 200 años, el cual implicó la elaboración del instrumento adecuado (termómetro), parámetros conceptuales (establecimiento de los puntos fijos) y construcción de aparatos matemáticos-teóricos (termodinámica, teorías del calor/energía) para obtener la representación científica matemática en forma de ecuación.

En este enfoque de la medición como producto van Fraassen desarrolla y caracteriza tres tipos de modelos (teórico, de datos y de superficie), para mostrar cómo se construye la representación científica. De Patrick Suppes toma la construcción de modelos, pues los modelos de datos construidos de manera experimental le ofrecen al científico teórico estructuras construidas cuidadosamente de la selección de datos.

La representación científica, según el autor, tiene tres fases (teoría-fenómeno-apariencias) que establecen los tres niveles de representación en las ciencias físicas. En la teoría se postula una realidad teóricamente (micro estructuras, fuerzas, campos, estructuras espacio-temporales globales); en el fenómeno se establece aquello que es el fenómeno-observable (macro objetos, movimiento, cuerpos visibles y tangibles), y en las apariencias se postula lo que apa- 
rece del fenómeno desde la medición (resultados/productos de la medición, "cómo las cosas lucen" en el contexto observacional). Van Fraassen remarca que no es lo mismo que las teorías salven los fenómenos a que las teorías estén en concordancia con los resultados experimentales y observacionales; cuando las teorías están en concordancia, representan las apariencias de los fenómenos tal y como aparecen en los resultados experimentales-observacionales. Por medio de la medición científica, la ciencia representa cómo lucen los fenómenos, no cómo son: la medición representa científicamente las apariencias.

Para van Fraassen, el fenómeno es aquello que aparece ante nuestros sentidos y por tanto es observable. La medición científica localiza y representa los fenómenos observables. Aunque esté consciente de muchos casos en la ciencia donde la medición se realiza en fenómenos/entidades/cantidades no observables (fenómenos detectados, predichos y/o deducidos), su idea de la medición científica se centra en medir lo observable. Su caracterización del modelo de datos es un registro de los resultados de medición que con frecuencia se expresan en estructuras matemáticas abstractas. Este modelo se construye a partir de la selección de datos de acuerdo con determinados criterios, es decir, es construido por la colección o sumario de datos. Por medio de la construcción de dicho modelo el científico experimental le ofrece al científico teórico pequeñas estructuras relacionales construidas de manera cuidadosa por datos seleccionados. En esta fase los resultados de los procedimientos de medición son registrados en los modelos de datos, los cuales se expresan en estructuras matemáticas y abstractas. El fenómeno observable aparece por primera vez en los resultados específicos de medición o en un gran conjunto de mediciones; el modelo de datos se construye con estos resultados individuales obtenidos por la medición. La construcción de los modelos de datos es la descripción selectiva y relevante del fenómeno por un agente 
que conoce la teoría requerida, lo que posteriormente posibilitará la representación del fenómeno, proceso o evento. Un modelo de datos particular es relevante porque fue construido bajo las bases de los resultados reunidos y seleccionados por un criterio específico de relevancia, en ciertas ocasiones, en un entorno experimental u observacional diseñado para ese propósito.

La tradición semanticista interpreta a este tipo de modelos como aquellos que organizan los datos brutos en una forma estándar; esto permite comparar los datos producidos de forma experimental con las predicciones de los modelos teóricos (por ejemplo, cómo analizar los valores individuales de medición de la temperatura del plomo en estadística). Los modelos de datos sólo pueden usarse después de aplicarles alguna técnica de análisis y no tienen nada que ver con cualquier tipo de afirmación teórica que pretenda modelar un fenómeno determinado (Bailer-Jones, 2009: 171).

Van Frassen introduce un modelo que no aparece en la tradición de interpretación semanticista, el cual interpreta a las teorías como familias de modelos, y lo denomina modelo de superficie, ya que integra al modelo de datos más las observaciones recolectadas que fueron acumuladas con labor a lo largo de espacios de tiempo prolongados, como el caso de las astronómicas. También, extrapola los patrones del modelo de datos en algo más fino de lo que los aparatos pueden registrar, puesto que se construye con los datos previamente obtenidos, organizados y analizados en el modelo de datos, para combinarlos con una colección de observaciones registradas. La estructura de la superficie de dicho modelo embona (se ajusta, se adecua) en un modelo teórico cuya estructura es matemática. En el modelo de superficie hay un isomorfismo que relaciona dos entidades abstractas: una entidad empírica (estructura de una teoría dada que describe los objetos observables relevantes de los objetos, procesos o eventos) y el modelo de superficie (la estructura matemática que representa los datos -las apariencias 
registradas por la medición- que han sido obtenidas de manera empírica). Por otra parte, el modelo de superficie le sirve para resolver los problemas que surgieron respecto del isomorfismo en $\mathrm{La}$ imagen cientifica, al pretender establecer un isomorfismo entre una entidad observable concreta y una entidad abstracta matemática. Este tipo de modelo apoya su idea de medición científica.

El modelo de superficie integra las condiciones observables en términos de las mediciones que se realizan y en los posibles resultados que se obtendrán después de realizar las mediciones y tiene la particularidad de ofrecer una representación construida en el laboratorio o en el observatorio, según el autor. Esto no quiere decir que van Frassen no tenga una visión de la misma situación al especificar cómo puede representarse por medio de un modelo teórico; para él éste se puede especificar en un conjunto o familia de magnitudes físicas (que para el filósofo son observables), las cuales están en un rango de valores posibles (que tienen la posibilidad de tener un valor), un conjunto de estados de las magnitudes $\mathrm{y}$ una función que relaciona ambos conjuntos.

Van Fraassen no define al modelo teórico y se apoya en la postura semanticista que lo define como aquel que pretende capturar el fenómeno al proveer una descripción completa del mismo, la cual incluye los factores que sobresalen y resultan importantes para constituir dicho fenómeno. Por ejemplo, mapas que muestran la distribución de un objeto y que se usan para hacer tests acerca de un fenómeno expresado en un modelo teórico del fenómeno, como cuando se examina si las fuentes dobles de radio tienen uno o dos reactores (jets en inglés) o si los lóbulos (lobes en inglés) de una fuente de radio contienen más plasma viejo que los hotspots en las imágenes obtenidas de las galaxias radiales (Bailer-Jones, 2009: 172 y 173). 
De igual manera, los modelos teóricos los utiliza, en primer lugar para ajustar ${ }^{1}$ tanto los fenómenos observados como los posibles observables. A partir de que la descripción de estos fenómenos es hecha en la práctica por medio de modelos -sean "de datos" o "de superficie”- el requerimiento para que éstos se ajusten a los fenómenos es el siguiente: los modelos de datos o superficies ${ }^{2}$ deben idealmente ser ajustados/incrustados de manera isomórfica en los modelos teóricos. Éste es el criterio que van Fraassen utiliza para determinar la representación de un fenómeno.

Tomando en cuenta lo anterior, sostengo que la defensa de la postura empirista de van Fraassen respecto a la medición sólo enfatiza el aspecto teórico de la misma no refuerza el componente empírico de la misma. Entender a la medición como proceso permitiría ver en los casos particulares de representaciones científicas el cómo interactúan la teoría, el agente, el instrumento y la matriz experimental. Esta visión del proceso permite ver cómo la teoría y la experimentación se interrelacionan, y es en esta interrelación donde se pueden identificar los aspectos empíricos en el proceso de medir. Si bien en un inicio el autor resalta la importancia de la experimentación en la concepción de la medición como un conjunto, su caracterización de la misma es sólo como producto: como representación. ${ }^{3} \mathrm{El}$ análisis que realiza en torno a la medición se

${ }^{1}$ Van Fraassen define ajustar como embedding (en inglés), que significa mostrar un isomorfismo a partes seleccionadas de esos modelos. Que un fenómeno se ajuste a un modelo significa que sea isomórfico a una parte de ese modelo. Es así como el fenómeno y el modelo relevante tienen la misma estructura (van Fraassen, 2008: 247).

${ }^{2}$ Como ya se mencionó el isomorfismo se da entre subestructuras empíricas y el modelo de superficie, aunque el modelo de datos se presupone integrado al modelo de superficie.

${ }^{3}$ Si bien Van Fraassen considera el ejemplo de la temperatura en Scientific Representation: Paradoxes of Perspective (2008), su análisis se enfoca exclusivamente en resaltar los aspectos teóricos de la medición y sus productos finales: las re- 
centra principalmente en la teoría, el agente y el aparato, así como la manera en que estos tres componentes realizan mediciones científicas y producen representaciones a través de modelos.

Las teorías representacionalistas de la medición enfatizan el aspecto formal de la medición en donde únicamente los productos de la medición son relevantes, suponen que vía estructuras se puede relacionar un objeto concreto, que es parte de un fenómeno que se enmarca dentro de una teoría, con elementos abstractos que frecuentemente se expresan en ecuaciones matemáticas. Un empirismo como el de van Fraassen no está mostrando el carácter empírico de la medición sino un supuesto realista que es utilizado para defender un empirismo en la ciencia y en la medición.

Una visión más amplia de la medición científica reconocerá que las teorías representacionalistas no son las únicas formas de medir en la ciencia, por ejemplo, la escala Mohs para medir la dureza de un objeto, en la que el principal uso de esta medida es la identificación mineral (Adams, 1966).

\section{Objecciones a las interpretaciones representacionalistas de la medición}

La teoría representacionalista de la medición surge a principios de la década de1900 y se concretiza en el primer volumen de Foundations of Measurement (1971), estableciendo los métodos, operaciones, parámetros y criterios para medir en la ciencia desde supuestos realistas. Uno de los componentes centrales de esta tradición parte de un cierto realismo ingenuo, el cual acepta que el mundo es de

presentaciones científicas. No profundiza al interior de un proceso de medición determinado para analizar cómo los componentes teóricos y los experimentales se interrelacionan a lo largo de un periodo de tiempo para finalmente lograr obtener una medición que pueda ser matemáticamente representable en un modelo y/o ecuación. 
la forma en la que corresponden las representaciones que se han construido con los datos precisos y los cálculos matemáticos obtenidos por la medición. En este contexto, el éxito en la ciencia ha sido valorado en torno a la exactitud de las representaciones que del mundo físico ha realizado; la exactitud se puede entender como la correspondencia entre las representaciones y ciertas regiones del mundo físico. Esta manera de considerar el éxito en la ciencia y la exactitud de las representaciones científicas es una posición realista científica ingenua.

Otra de sus principales características es la presuposición de un isomorfismo (u homomorfismo) entre algún sistema empírico relacional y uno relacional. Debido a dicha presuposición, cierta función representativa es generalmente asignada al mapeo numérico. Es decir, la teoría representacionalista de la medición requiere: afirmar que los números se asignan a los objetos empíricos y especificar que las relaciones definitivas entre números y operaciones (con números) correspondan a ciertas relaciones entre éstos y las operaciones con ellos. Las relaciones definitivas dependen a su vez de otras relaciones entre números y operaciones, y ambas deben ser especificadas. Así también, esta teoría debe justificar de manera teórica las condiciones en las cuales la asignación de números a objetos empíricos es posible, además de que dicha asignación sea empíricamente significativa y realizable de forma operacional.

Para justificar la existencia de la correlación entre un sistema empírico y un sistema relacional numérico, entre hechos empíricos y expresiones numéricas, con frecuencia se utilizan modelos empíricos que se ajusten al sistema numérico relacional; cuando se obtiene un ajuste entre todos los elementos de un sistema empírico y todos los elementos del sistema relacional numérico, la representación del hecho empírico es isomórfica. El análisis de Karel Berka (1983) sostiene que las teorías representacionalistas de la medición requieren justificar la relación entre hechos y expresiones 
numéricas; su mayor problema es obtener mejores criterios que justifiquen dicha relación en términos de isomorfismo. Aunque la teoría representacionalista de la medición sea una teoría robusta actual, no refleja todas las formas y métodos de medición en la historia de la ciencia.

El principal análisis en torno a los supuestos realistas en las teorías representacionalistas de la medición es de Joel Michell (2005), su enfoque realista pretende evitar las idealizaciones al no considerar a los números como entidades abstractas (entidades existentes fuera del espacio y el tiempo). Su postura principal radica en no emplear entidades (números), las cuales están relacionadas externamente a características de situaciones empíricas por convenciones humanas. La teoría realista de la medición pretende explicarla desde un enfoque naturalista en el que el entendimiento de la naturaleza es espacio-temporal. Sin embargo, la propuesta de Michell no parece ofrecer una postura alternativa real a la teoría representacionalista de la medición, pero, debido a que el autor es un especialista de la tradición en la medición científica, su análisis resulta pertinente para este texto.

El argumento de Michell parte de la idea de realismo, en la cual los métodos científicos promueven la adquisición de conocimiento acerca de la estructura y las maneras de operar de los distintos sistemas naturales que se investigan. La medición es uno de los métodos científicos más importantes; por ello, le resulta sorprendente que no existan interpretaciones realistas en la literatura metrológica. Michell asocia el realismo en la ciencia con un concepto literal de verdad que nos compromete con la existencia de las cosas independientemente de la observación. El autor hace una distinción entre realismo metafísico y realismo epistemológico: el primero sostiene la existencia de un mundo objetivo estructurado de manera espacio-temporal (existencia del mundo independiente del sujeto), mientras que el segundo afirma que las cosas son ló- 
gicamente independientes de la observación (conocimiento de las cosas por la lógica y la matemática). Además, sostiene que la visión en la cual se considera que los números son relaciones localizadas espacio-temporalmente se compromete con una visión realista del número. Para Michell, los defensores del concepto realista de verdad interpretan los números reales como razones.

Los compromisos ontológicos de entender a la medición desde el concepto realista de verdad ${ }^{4}$ no se dan sólo con la existencia lógicamente independiente de las cosas en el espacio y en el tiempo, sino también con la existencia de las propiedades y relaciones estructuradas de forma cuantitativa y con la existencia de los números reales, entendidos como las relaciones de razón entre niveles específicos de dichos atributos. La medición es el intento de estimar la razón entre dos instancias de un atributo cuantitativo: la primera es la magnitud medida y la segunda la unidad conocida.

La conclusión de Michell es que la medición científica en general se basa en la hipótesis de que algún atributo es cuantitativo. En cualquier ciencia esta hipótesis afirma que ciertas condiciones empíricas se obtienen y por esto se descartan otras posibilidades. El método científico de indagación crítica, según el cual las hipótesis sólo se aceptan siguiendo serios intentos que las ponen a prueba para ofrecer evidencia a su favor, puede aplicar con cualquier hipótesis. El argumento de Michell sostiene que tradicionalmente las teorías representacionalistas de la medición han interpretado a los números reales como razones, y han establecido así compromisos realistas que se adquieren cuando es aceptada la hipótesis de que un atributo es cuantitativo; es decir, que además de la aceptación de dicha hipótesis - como parte del mismo paquete teórico- se acepta también que diferentes magnitudes del atributo mantienen rela-

${ }^{4}$ Michell no clarifica qué teoría de la verdad está considerando. La noción realista de verdad que menciona pretende explicitar los compromisos ontológicos respecto a la existencia de las cosas y de las entidades matemáticas. 
ciones de razón; porque dichas relaciones son instancias de números reales. Por tanto, los números reales se consideran localizados en el contexto empírico de la medición como aspectos intrínsecos. Cuando los científicos se proponen idear métodos prácticos para medir, lo que pretenden estimar es precisamente estos números reales. Para Michell, la manera de medir no es particular en relación a otros tipos de procedimientos en la ciencia; no hay nada que haga a la medición diferente en principio a cualquier otra actividad científica. El análisis de Michell permite considerar otros aspectos, además de los teóricos, en un proceso de medición que no se enfoque únicamente en una interpretación realista de las matemáticas que privilegie los componentes teóricos de la medición. Si bien el autor no propone una alternativa a la interpretación realista, sus críticas sirven para comprender los fundamentos realistas en los que descansa la tradición de las teorías representacionalistas de la medición. Por otro lado, su argumento en torno a los números reales no parece del todo sólido porque igualmente podría sostenerse una interpretación realista de la razón en la medición.

Otro autor que ha analizado problemáticas en la interpretación representacionalista de la medición es David Sherry (2011), quien afirma que la teoría de la medición analiza los procedimientos usados para derivar mediciones cuantitativas de observaciones cualitativas. La medición desde un enfoque representacionalista prescribe la estructura que un sistema de observaciones cualitativas debe tener para que las relaciones numéricas puedan usarse -ya sea para representar o describir aquellas observaciones-, de forma tal que permita realizar inferencias matemáticas acerca de las situaciones empíricas en las que las observaciones se trazaron. También, señala que la representación difiere de la descripción: cuando se prescribe la estructura no se hace una distinción entre ambas porque lo que importa es realizar las inferencias matemáticas; por lo tanto, 
las diferencias sólo se establecen en los desacuerdos en torno a los informes/resultados de la medición.

El autor sostiene que, por un lado, los empiristas consideran abstractos los resultados de la medición: representaciones numéricas de contenidos empíricos; por el otro, los realistas afirman que los resultados de la medición describen relaciones empíricas numéricas entre magnitudes (por ejemplo, niveles de un atributo). Del mismo modo, afirma que ninguna de las dos posturas es satisfactoria, porque ambas requieren que el mundo empírico tenga una estructura (estructura fija, que no cambia y es independiente de los sentidos); es decir, ambas posturas suponen o presuponen una estructura en el mundo empírico la cual está mucho más allá de lo que se puede acceder empíricamente. Para el autor, este requerimiento (que el mundo tenga una estructura exacta) sobrepasa los predicados empíricos, si consideramos que aquello que podemos decir del mundo está basado en nuestro contacto empírico con él.

Sherry sostiene que ni la formulación realista ni la empirista de la medición están vinculadas a la observación; ambas versiones privilegian la adaptación a sus axiomas en defensa de sus teoremas representacionalistas. Dichos axiomas involucran conceptos exactos y estructuras cuasi empíricas. De este modo, los tests empíricos -tanto para los realistas como los empiristas-, implican identificar conceptos de acuerdo con la exactitud, noción que no es identificable de manera empírica. Y como no se pueden observar instancias de conceptos exactos, las bases para su identificación son pragmáticas.

Según el autor, la historia de la medición de la temperatura sugiere un punto medio entre adoptar un programa de investigación dictado por estas teorías de la medición o simplemente abandonar la tarea de la cuantificación. El caso de la temperatura ofrece un largo proceso de medición que involucró tanto un acceso empírico experimental como un desarrollo conceptual. Para Sherry, la afir- 
mación de Michell que sostiene que los realistas apoyan una teoría representacionalista de la medición tradicional -en la cual los números tienen una existencia real en el mundo-y que los empiristas consideran a los números como construcciones de entidades abstractas hechas por el hombre desprovistas de contenido empírico, reafirma que ambas posturas de la medición no están vinculadas a la observación y traspasan aquello a lo que puede accederse empíricamente.

La conclusión de Sherry es que la teoría representacionalista de la medición bien puede enmarcarse en términos de atributos en lugar de objetos o eventos; los atributos estarían en una dependencia empírica (se puede acceder a ellos empíricamente), en cambio los objetos o eventos suponen una estructura.

Los análisis tanto de Michell como de Sherry permiten apreciar que la medición científica está fincada en una tradición realista que predetermina en sus interpretaciones un andamiaje estructural construido a través de modelos y relaciones isomórficas que privilegian los aspectos teóricos de la medición más que los procesos empírico-experimentales.

Si bien van Fraassen defiende un empirismo en la ciencia, su propuesta en torno a la medición científica está basada en una tradición representacionalista que asume compromisos realistas. Aun cuando en el texto "Modeling and Measurement: The Criterion of Empirical Grounding” (2012), que es posterior a Scientific Representation: Paradoxes of Perspective (2008), expone ejemplos experimentales de medición, su postura sigue fincada en el componente teórico de la medición. Debido a lo anterior, y considerando el ejemplo citado por Sherry del proceso de medición de la temperatura que involucró tanto un acceso empírico experimental como un desarrollo conceptual, considero importante ejemplificar la relación teórica-experimental de los procesos de medición con el caso de la medición de la velocidad de la luz propuesto por Al- 
bert A. Michelson (1852-1931) en 1877, para apoyar así la idea de "iteración epistémica", que es una interrelación entre teoría y experimentación en la que ambas se retroalimentan en un proceso de modificación constante.

\section{La medición como proceso en el experimento de medi- ción de la velocidad de la luz}

En noviembre de 1877, Albert A. Michelson (1852-1931) realizó un experimento para medir la velocidad de la luz blanca mientras viaja entre dos puntos relativamente inmóviles en el vacío, con el cual obtuvo medidas más precisas basadas en una versión mejorada del espejo rotatorio de León Foucault (1819-1868). El espejo rotatorio sustituyó la rueda dentada ${ }^{5}$ elaborada por Fizeau (1819-1896), quien produjo la primera determinación terrestre de la velocidad de la luz en 1849 con una innovación al colocar un espejo fijo cuya superficie era perpendicular al rayo de luz que provenía de la fuente de luz; con dicha innovación, el rayo de luz se reflejaba directamente desde su origen y las mediciones eliminaban cualquier tipo de variación en la percepción humana (al suplir el espejo por el sujeto observador).

El experimento de Michelson mejora tanto el experimento de Foucault como el de Fizeau. Respecto a este último, la limitante estaba en el tiempo de la desaparición total de la luz, porque era incierto el momento en que desaparecía de una ranura y aparecía en la siguiente. La principal objeción de Michelson al experimento de Foucault era que la cantidad del desplazamiento entre el rayo

${ }^{5}$ Rueda dentada de rotación rápida que se usaba para medir la velocidad de la luz ajustando la rotación hasta que pasara por una de las ranuras y la reflejara desde un espejo distante para que volviera a pasar la siguiente ranura. Con este método se tenía la velocidad de rotación de la rueda y la distancia recorrida (ida y regreso del rayo de luz) para obtener la velocidad de la luz. 
del origen y el reflectado era muy pequeña y, por tanto, difícil de medir con precisión.

El análisis y la explicación de MacKay y Oldford (2000) plantea que del experimento de Michelson puede extraerse un proceso que consta de cinco pasos interrelacionados entre ellos, en donde cada paso permite hacer ajustes, adecuaciones, ya sea para pasos subsecuentes o antecedentes. Esquemáticamente, este proceso puede organizarse en las siguientes etapas: el problema, el plan, los datos, el análisis y la conclusión.

El problema: en 1879, Michelson se propuso determinar la velocidad de la luz blanca mientras viaja entre dos puntos relativamente inmóviles en el vacío. La unidad fue la transmisión de dicha luz entre la fuente y el destino, ambos localizados en el vacío. En esta fase se establecen los procesos para el objetivo (target), teniendo la teoría de fondo que considera que la velocidad de la luz blanca es constante en el vacío, así que no existen variables en el valor de respuesta. El problema en este caso es descriptivo pues el propósito es estimar el promedio de la velocidad de la luz.

El plan: en esta fase se elabora un procedimiento para la obtención y análisis de los datos. Michelson midió en cada determinación las respuestas de las variables para calcular la velocidad de la luz. Anticipó que el intento de medir la velocidad de la luz en el aire resultaría en un error, el cual planeó corregir usando un factor basado en el indicador de la refracción del aire; esto requirió de sus conocimientos contextuales en torno a teorías previas. El error de medición lo caracterizó como la diferencia entre el valor de la variable determinado por el proceso de medición y el valor de la unidad de la muestra (la unidad en este caso es una transmisión de luz entre la fuente y el destino, ambos localizados en el vacío). Las propiedades del proceso de medición se definen en términos de medir repetidamente la misma unidad de estudio. Existen dos sesgos en este proceso: un atributo del objetivo (target) en el proceso 
de medición, que describe sistemáticamente un error de medición, y la variabilidad de medición, un atributo en el proceso que describe el cambio en el error de medición de una determinación a la siguiente.

Los datos: en esta fase se incluyen la ejecución del plan y la verificación de la calidad de los datos para el análisis. El primer conjunto de mediciones realizadas por Michelson se realizó con luz eléctrica; este método fue abandonado y comenzó a utilizar luz natural al observar que la imagen con luz artificial no era tan nítida. Dichas modificaciones sugieren que el monitoreo de los datos lo llevó a realizar cambios en el plan. El examen de los datos procura la consistencia interna de los mismos como un todo; los datos son examinados para encontrar patrones o en su defecto rasgos inesperados.

El análisis: aquí se utilizan los datos y la información obtenida por medio del plan para tratar las preguntas formuladas. Con frecuencia se construye un modelo para el plan y los datos, los cuales se ajustan y evalúa el modelo para usar un modelo final que permita resolver el problema. En el caso de Michelson, el análisis lo limitó a calcular el promedio de 100 velocidades medidas en el aire, realizar una colección de números y estimar posibles errores; aunque, tampoco consideró hacer comparaciones entre las mediciones realizadas en el día y la noche.

La conclusión: en su estudio Michelson concluyó haber reportado que la velocidad de la luz (kilómetros por segundo) en el vacío es de 299,944 \pm 51 . Posteriormente, discutió algunas objeciones, entre ellas: la incertidumbre en las leyes de reflexión y refracción en un medio en rotación rápida, el retraso causado por la reflexión, las imperfecciones en los lentes, las variaciones periódicas en la fricción de los pivotes del espejo rotatorio y el cambio en la velocidad de rotación. Para cada una de las objeciones, Michelson refiere al plan y al modelo de valoración para mostrar que éstas pueden 
tener un pequeño efecto en la estimación de la velocidad de la luz. El ejemplo de Michelson permite ver todos los pasos y ajustes realizados en un proceso de medición en los que se interrelacionan el sujeto (agente), los instrumentos experimentales y la(s) teoría(s). Es un proceso que consta de varias etapas hasta lograr el objetivo de medir la velocidad de la luz en determinadas condiciones.

Los procesos de medición científica son en realidad procesos dinámicos autocorregibles de iteración y retroalimentación, los cuales inician históricamente a partir de un estado específico de conocimientos que se va modificando conforme se desarrollan etapas sucesivas de investigación, tal y como lo ha establecido Chang (Chang, 2006). La sucesión de estas etapas conforman el proceso histórico completo de medición que finalmente llega a determinar el valor específico de la magnitud física que inicialmente se buscaba. Cada una de dichas etapas tiene rasgos diferentes que pueden ir desde obtener nueva información y resolver problemas específicos, hasta calcular valores desconocidos y descubrir errores, etc. Como lo vimos en el caso de la medición de la velocidad de la luz, cada una de las etapas que estuvieron involucradas en la investigación de Michelson tuvieron los rasgos señalados y la información y/o los problemas resueltos en etapas posteriores, donde modificaba, ajustaba y/o corregía los resultados de las etapas anteriores, lo cual genera un proceso de continuo ajuste a través de las mismas. ${ }^{6} \mathrm{La}$ idea central de este tipo de proceso es que las etapas posteriores tienen como una de sus finalidades epistémicas fundamentales el revisar y en su caso corregir los resultados de las etapas anteriores y reintegrarlos en nuevos cálculos actualizados. Nótese que a lo largo de este proceso pueden eliminarse resultados de etapas anteriores

${ }^{6}$ Como ya mencioné al inicio, este tipo de proceso es denominado por Hasok Chang (2006) de iteración epistémica: "un proceso en el cual etapas sucesivas de conocimiento, cada una edificada sobre las anteriores, son creadas con el fin de mejorar el logro de ciertas metas epistémicas" (Chang, 2006: 45). 
pero lo que persiste a lo largo de todo el proceso es el objetivo a medir. Al inicio de este proceso el científico sabe qué se quiere medir (target) pero lo que no puede saber es la naturaleza de los problemas que enfrentrará en cada una de las etapas y mucho menos saber por adelantado cuáles serán éstas.

A lo largo de su análisis en torno a la medición, van Fraassen resalta la relevancia de considerar a la medición científica como un proceso que produce representaciones, sin embargo, no muestra con algún ejemplo el cómo podría apreciarse a la medición como un proceso y se enfoca en los productos finales de ésta: las representaciones científicas.

La medición científica es un complejo proceso iterativo que está entre la representación y la intervención al involucrar relaciones de retroalimentación entre la teoría y la experimentación que produce representaciones.

\section{Bibliografía}

Adams, Ernest W., 1966, "On the nature and Purpose of Measurement”, Synthese, vol. 16, núm. 2, pp. 125-169.

Bailer-Jones, Daniel, 2009, Scientific Models in Philosophy of Science, Pittsburgh, University of Pittsburgh Press.

Balzer, Wolfgang,1992, "The structuralist view of measurement: An extension of received measurement theories", en Philosophical and Foundational Issues in Measurement Theory, Nueva Jersey, Lawrence Erlbaum Associates Publishers, pp. 93-117.

Batitsky, Vadim, 1998, "Empiricism and the myth of fundamental measurement", Synthese, vol. 116, núm. 1, pp. 51-73.

Berka, Karel, 1983, Measurement, its Concepts, Theories and Problems, Holanda, D. Reidel Publishing Company. 
Chang, Hasok, 2004, Inventing Temperature, Oxford, Oxford University Press.

Ellis, B., 1968, Basic Concepts of Measurement, Londres, Cambridge University Press.

Hacking, Ian, 1983, Representing and Intervening: Introductory Topics in the Philosophy of Natural Science, Cambridge, Cambridge University Press.

, 1988, "Philosophers of experiment", PSA: Proceedings of the Biennial Meeting of the Philosophy of Science Association, vol. 2: Symposia and Invited Papers, Chicago, The University of Chicago Press, pp. 147-156.

Krantz, D.H., Luce R. D. y Suppes P., 1971, Foundations of Measurement, Vol. I: Additive and polynomial representations, Nueva York, Academic Press.

MacKay, R. J. y Oldford R. W., 2000, "Scientific Method, Statistical Method and the Speed of Light", Statistical Science, vol. 15, núm. 3, pp. 254-278.

Michell, Joel, 2005, “The logic of measurement: A realist overview”, Measurement, vol. 38, pp. 285-294.

, 2007, "Measurement" en Handbook of the Philosophy of Science, Philosophy of Anthropology and Sociology, Holanda, Elsevier.

, 1997, 'Bertrand Russell's 1897 critique of the traditional theory of measurement", Synthese, vol.110, pp. 257-276.

Michelson, Albert Abraham y Edward, W., Morley, 1887, "On the relative motion of the earth and the luminiferous ether", The American Journal of Science, vol. XXXIV, núm. 203, pp. 333-345.

Michelson, Albert Abraham, 1920, "A modification of the revolving mirror method for measuring the velocity of light", Pro- 
ceedings of the National Academy of Sciences of the United States of America, vol. 6, núm. 8, pp. 476-477.

, 1928, "Experimental determination of the velocity of light”, The Scientific Monthly, vol. 27, núm. 6, pp. 562-565.

Michelson, Albert Abraham, Pease F. G. y Pearson F., 1935, "Measurement of the velocity of light in a partial vacuum", Science, nueva serie, vol. 81, núm. 2091, pp. 100-101.

Nagel, Ernest, 1931, "Measurement”, Erkenntnis, vol. 2, pp. 313335.

Russell, B., 1903, Principles of Mathematics, Londres, Bradford and Dickens.

Sherry, David, 2011, "Thermoscopes, Thermometers, and the Foundations of Measurement", Studies in History and Philosophy of Science, vol. 42, pp. 509-524.

Stevens, S. S., 1946, "On the theory of scales of measurement", Science, nueva serie, vol. 103, núm. 2684, pp. 677-680.

Suppes, P. y Joseph L. Zinnes, 1963, "Basic Measurement Theory”, en Luce, Bush y Galanter (eds.), Handbook of Mathematical Psycology, vol. I, Nueva York, John Wiley and sons.

Van Fraassen, Bas C., 1980, The Scientific Image, Oxford, Oxford University Press.

, 1996, La imagen cientifica, Paidós/UNAM, México.

, 2008, Scientific Representation: Paradoxes of Perspective, Oxford, Clarendon press/Oxford University Press.

, 2012, "Modeling and Measurement: The Criterion of Empirical Grounding", Philosophy of Science, vol. 79, núm. 5, pp. 773-784. 